\title{
High order optimal feedback control of space trajectories with bounded control
}

\author{
P. Di Lizia*, R. Armellin, A. Morselli, F. Bernelli-Zazzera \\ Politecnico di Milano, Dipartimento di Ingegneria Aerospaziale, Via La Masa 34, 20156 \\ Milano, Italy
}

\begin{abstract}
Optimal feedback control is classically based on linear approximations, whose accuracy drops off rapidly in highly nonlinear dynamics. Several nonlinear optimal feedback control strategies have appeared in recent years. Among them, differential algebraic techniques have been used to tackle nonlinearities by expanding the solution of the optimal control problem about a reference trajectory and reducing the computation of optimal feedback control laws to the evaluation of high order polynomials. However, the resulting high order method could not handle control saturation constraints, which remain a critical facet of nonlinear optimal feedback control. This work introduces the management of saturating actuators in the differential algebraic method. More specifically, the constraints are included in the optimal control problem formulation and differential algebra is used to expand the associated optimal bang-bang solution with respect to initial and terminal conditions. Optimal feedback control laws for thrust direction and switching times are again computed by evaluating the resulting polynomials. Illustrative applications are
\end{abstract}

\footnotetext{
${ }^{*}$ Corresponding author. E-mail: pierluigi.dilizia@polimi.it
} 
presented in the frame of the optimal low-thrust transfer to asteroid 1996 $\mathrm{FG}_{3}$.

Keywords: Low-thrust transfers, Optimal control, High order methods, Saturating actuators

\section{Introduction}

Continuous-thrust orbit transfers are designed by solving an optimal control problem that minimizes fuel consumption while satisfying mission constraints. The optimal control problem is solved in nominal conditions: at the design stage, the dynamics modeling is supposed to exactly represent the reality. Unfortunately, the accuracy of the dynamical models is affected by the representation of all possible perturbations, which is difficult to produce. Uncertainty does not owe to dynamics perturbations only. Several spacerelated applications involve the solution of optimal control problems with uncertain constraints or terminal conditions [1]. A typical example is the problem of targeting an asteroid [2] (e.g., during a deflection mission). The orbital parameters of the target asteroid are known only to a given precision and, thus, its position and velocity are accurately identified quite late in real scenarios. This draws the designer towards the development of optimal feedback control strategies to achieve constraint satisfaction and optimality in presence of errors on both the initial spacecraft and the final target states.

Classical optimal feedback control strategies are based on linear approximations, whose main advantage is the simplification of the problem. However, their accuracy drops off rapidly for increasing errors and decreasing control frequencies in highly nonlinear dynamics. Thus, nonlinear optimal 
feedback control has gained particular interest in recent years, and several strategies have appeared to tackle nonlinearities. Nonlinear optimal feedback control laws can be rigorously obtained from the solution to the HamiltonJacobi-Bellman (HJB) equation [3]. Unfortunately, solving the HJB equation is very intricate in practical problems. This motivated research for approximated methods that are able to supply suboptimal laws for the closed-loop control of nonlinear systems.

In the infinite horizon regulator problem, the time variable does not explicitly appear in the optimal feedback control. Several approaches have appeared to take advantage of this simplification and to obtain numerical approximations of the exact solution. Durbeck [4] provided a first method to generate an approximation to the minimum performance functional of the optimal feedback control problem, showing its convergence properties and its application to design near optimal control laws, determining regions of stability, and defining bounds associated with the system performance. More recently, Beard et al. [5] tried to reduce the computational burden of finiteelements and finite-difference methods applied to solving HJB equation by using a Galerkin's spectral method. One of the highly promising and rapidly emerging methodologies for designing nonlinear optimal controllers in the infinite horizon formulation is the state-dependent Riccati equation (SDRE) approach, which was originally proposed by Pearson [6] and Burghart [7] and then described in details by Cloutier, Hammett and Beeler [8]. This approach involves manipulating the governing dynamic equations into a pseudo-linear non-unique form in which system matrices are given as a function of the current state and minimizing a quadratic-like performance index. An algebraic 
Riccati equation using the system matrices is then solved repetitively online to give the optimal control law. Thus, the SDRE approach might turn out to be computationally expensive when the solution of the Riccati equation is not properly managed. This might prevent its use for real-time optimal control.

Due to the explicit dependence of the HJB equation on time, the finite horizon targeting problem tends to be more difficult to solve than the infinite horizon problem [9]. Thus, relatively few results exist in the literature. Remarkable results were obtained by Fax et al. [10] in the framework of the optimal control of time-scalable systems. The time-scaling property allows to convert the HJB equation to a purely spatial PDE. The PDE is then solved to compute the value function at a fixed time, which can be properly scaled to find the value function at any point in time. Following the "pseudo-linear" approach of SDRE, Cimen and Banks [11] introduced a sub-optimal method to find time-varying feedback controllers for nonlinear systems, which is based on the recursive solution of an approximating sequence of Riccati equations.

An alternative approach was recently proposed by Park and Scheeres [12], which relies on the theory of canonical transformations and their generating functions for Hamiltonian systems. More specifically, canonical transformations are able to solve boundary value problems between Hamiltonian coordinates and momenta for a single flow field. Thus, based on the reduction of the optimal control problem to an equivalent boundary value problem, they can be effectively used to solve the optimal control problem analytically as a function of the boundary conditions, which is instrumental to optimal feedback control. The main difficulty of this approach is finding the generating 
functions via the solution of the Hamilton-Jacobi equation. This problem was solved in [12] by expanding the generating function in power series of its arguments.

Unfortunately, limited research has been devoted to develop efficient nonlinear techniques handling control saturation. The introduction of control bounds in the SDRE approach was addressed by Mracek and Cloutier [13, 14]. More specifically, they transformed the nonlinear regulator problem with bounded control into a near-equivalent problem with the form of a nonlinear regulator problem to avoid singularities. A solution procedure was introduced by Park and Scheeres [15] for the generating function method to accommodate control constraints, which is based on the fact that the optimal cost function can be related to the generating functions even in presence of control bounds.

A high order method based on the use of differential algebraic techniques is proposed in this work. Differential algebra (DA) serves the purpose of computing the derivatives of functions in a computer environment. More specifically, by substituting the classical implementation of real algebra with the implementation of a new algebra of Taylor polynomials, it expands any function $f$ of $v$ variables into its Taylor series up to an arbitrary order $n$ $[16,17]$. Following the reduction of the optimal control problem (OCP) to a two-point boundary value problem, DA techniques have been shown to enable the expansion of the OCP solution about a reference trajectory with respect to either initial or terminal conditions [18]. The computation of feedback control laws in relatively large neighborhoods of the reference trajectory is then reduced to the mere evaluation of high order polynomials. 
This work introduces the management of saturation constraints in the DA framework to define an high order optimal feedback control algorithm with saturating actuators. The control constraints are included in the OCP formulation and a minimum fuel mass low-thrust transfer problem is solved. A DA-based algorithm to compute arbitrary order expansions of the associated optimal bang-bang solution about reference trajectories with respect to initial and terminal conditions is presented. The computation of both optimal thrust direction and switching times for perturbed initial spacecraft and final target states is again reduced to the mere evaluation of high order polynomials.

The paper is organized as follows. After a brief introduction to DA techniques, the basic tool to compute high order expansions of the flow of ODEs is presented. Then, the optimal control problem and the numerical approach adopted to compute the reference trajectory are introduced. The description of the algorithm for the high order expansion of the OCP follows. The performances of the methods are assessed in the frame of an optimal low-thrust transfer to asteroid $1996 \mathrm{FG}_{3}$.

\section{Differential Algebra}

DA techniques find their origin in the attempt to solve analytical problems by an algebraic approach [16]. Historically, the treatment of functions in numerics has been based on the treatment of numbers, and the classical numerical algorithms are based on the mere evaluation of functions at specific points. DA techniques are based on the observation that it is possible to extract more information on a function rather than its mere values. The ba- 
sic idea is to bring the treatment of functions and the operations on them to the computer environment in a similar way as the treatment of real numbers. Referring to Figure 1, consider two real numbers $a$ and $b$. Their transformation into the floating point representation, $\bar{a}$ and $\bar{b}$ respectively, is performed to operate on them in a computer environment. Then, given any operation $x$ in the set of real numbers, an adjoint operation $\otimes$ is defined in the set of FP numbers such that the diagram in figure commutes. (The diagram commutes approximately in practice, due to truncation errors.) Consequently, transforming the real numbers $a$ and $b$ in their FP representation and operating on them in the set of FP numbers returns the same result as carrying out the operation in the set of real numbers and then transforming the achieved result in its FP representation. In a similar way, suppose two sufficiently regular functions $f$ and $g$ are given. In the framework of differential algebra, the computer operates on them using their Taylor series expansions, $F$ and $G$ respectively. Therefore, the transformation of real numbers in their FP representation is now substituted by the extraction of the Taylor expansions of $f$ and $g$. For each operation in the function space, an adjoint operation in the space of Taylor polynomials is defined such that the corresponding diagram commutes; i.e., extracting the Taylor expansions of $f$ and $g$ and operating on them in the function space returns the same result as operating on $f$ and $g$ in the original space and then computing the Taylor expansion of the resulting function.

The straightforward implementation of differential algebra in a computer enables the computation of the Taylor coefficients of a function up to a specified order $n$, along with the function evaluation, with a fixed amount of 

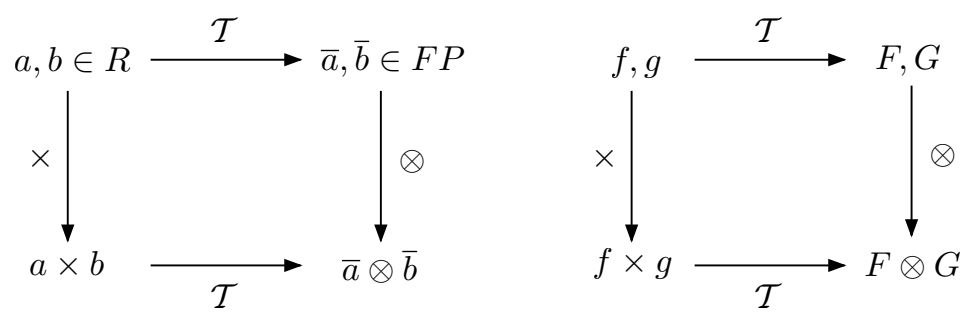

Figure 1: Analogy between the floating point representation of real numbers in a computer environment (left figure) and the introduction of the algebra of Taylor polynomials in the differential algebraic framework (right figure).

effort. The Taylor coefficients of order $n$ for sums and product of functions, as well as scalar products with reals, can be computed from those of summands and factors; therefore, the set of equivalence classes of functions can be endowed with well-defined operations, leading to a truncated power series algebra. Similarly to the algorithms for floating point arithmetic, the algorithm for functions followed, including methods to perform composition of functions, to invert them, to solve nonlinear systems explicitly, and to treat common elementary functions [16]. In addition to these algebraic operations, also the analytic operations of differentiation and integration are introduced, so finalizing the definition of the DA structure. The differential algebra sketched in this section was implemented by Berz and Makino in the software COSY-Infinity [17].

\section{High Order Expansion of ODE Flow}

The differential algebra introduced in the previous section allows to compute the derivatives of any function $f$ of $v$ variables up to an arbitrary order $n$, along with the function evaluation. This has an important consequence 
when the numerical integration of an ODE is performed by means of an arbitrary integration scheme. Without loss of generality, consider the scalar initial value problem

$$
\left\{\begin{array}{l}
\dot{x}=f(x) \\
x\left(t_{0}\right)=x_{0} .
\end{array}\right.
$$

Any explicit numerical integration scheme to solve the problem in Eq. 1 is based on algebraic operations, involving the evaluation of the ODE right hand side, $f(x)$, at several integration points.

Consider a perturbed initial condition $x_{0}+\delta x_{0}$, which is obtained by substituting the point initial condition, $x_{0}$, with the Taylor expansion of its identity function, $\left[x_{0}\right]=x_{0}+\delta x_{0}$. If all the operations of the numerical integration scheme are now carried out in the framework of differential algebra by operating on Taylor polynomials, the Taylor expansion of the solution with respect to the initial condition is obtained at each integration step. This can be better illustrated using the simple forward Euler scheme

$$
x_{k}=x_{k-1}+\Delta t \cdot f\left(x_{k-1}\right),
$$

where $\Delta t$ is the step size. The first integration step reads

$$
x_{1}=x_{0}+\Delta t \cdot f\left(x_{0}\right)
$$

Substituting $x_{0}$ with $\left[x_{0}\right]=x_{0}+\delta x_{0}$ in Eq. 3 yields

$$
\left[x_{1}\right]=\left[x_{0}\right]+\Delta t \cdot f\left(\left[x_{0}\right]\right) .
$$

The evaluation of $f\left(\left[x_{0}\right]\right)$ in the DA framework delivers its Taylor expansion $\mathcal{M}_{f}\left(\delta x_{0}\right)$ with respect to the initial condition about the reference point $x_{0}$. Then, by carrying out all the algebraic operations remaining in the right 
hand side of Eq. 4 in the same framework, the output of the first step is the Taylor expansion of the solution of the initial value problem with respect to the initial condition, $\left[x_{1}\right]=\mathcal{M}_{x_{1}}\left(\delta x_{0}\right)$. Similarly to Eq. 3 , the second step reads

$$
x_{2}=x_{1}+\Delta t \cdot f\left(x_{1}\right)
$$

Taking advantage of the first step, $x_{1}$ can be substituted with its Taylor expansion with respect to the initial condition, $\left[x_{1}\right]=\mathcal{M}_{x_{1}}\left(\delta x_{0}\right)$, which yields

$$
\left[x_{2}\right]=\left[x_{1}\right]+\Delta t \cdot f\left(\left[x_{1}\right]\right)=\mathcal{M}_{x_{1}}\left(\delta x_{0}\right)+\Delta t \cdot f\left(\mathcal{M}_{x_{1}}\left(\delta x_{0}\right)\right) .
$$

Once again, if all the operations in the right hand side of Eq. 6 are carried out in the DA framework, the output of the second step will be the Taylor expansion of the solution of the initial value problem at $t_{2}$ with respect to the initial condition, $\left[x_{2}\right]=\mathcal{M}_{x_{2}}\left(\delta x_{0}\right)$. The previous procedure can be repeated throughout the subsequent steps. The result at the final step is the $n$-th order Taylor expansion of the flow of the initial value problem at the final time $t_{f}$. Thus, the expansion of the flow of a dynamical system can be computed up to order $n$ with a fixed amount of effort.

\section{Optimal Control Problem}

The optimal transfer problem and the numerical approach adopted to compute its reference solution are now introduced. It is assumed that the spacecraft is subject to Sun's gravity and its own thrust $T$, only. Two control variables associated to spacecraft thrust are considered: the thrust ratio $u \in[0,1]$ and its direction $\boldsymbol{\alpha}$. The equation of the dynamics for the spacecraft position $\boldsymbol{r}$, velocity $\boldsymbol{v}$, and mass $m$, are 


$$
\left\{\begin{array}{l}
\dot{\boldsymbol{r}}=\boldsymbol{v} \\
\dot{\boldsymbol{v}}=-\frac{\mu}{r^{3}} \boldsymbol{r}+c_{1} \frac{u}{m} \boldsymbol{\alpha} \\
\dot{m}=-c_{2} u .
\end{array}\right.
$$

The two constants $c_{1}$ and $c_{2}$ in Eq. (7) are defined as

$$
\begin{aligned}
& c_{1}=T_{\max } \\
& c_{2}=\frac{T_{\max }}{I_{\mathrm{sp}} g_{0}},
\end{aligned}
$$

and $\mu$ is the gravitational constant of the Sun. The dynamics are normalized choosing the astronomical unit (AU), the initial mass of the spacecraft, and the velocity on a circular orbit of radius $1 \mathrm{AU}$ as reference units.

The goal is to minimize the propellant necessary to transfer the spacecraft between two fixed states in a given time of flight. This is achieved by defining an optimal control problem in which the objective function to be minimized is

$$
J=c_{2} \int_{t_{0}}^{t_{f}} u d t
$$

subject to the set of nonlinear constraints

$$
\left\{\begin{array}{l}
\boldsymbol{r}\left(t_{0}\right)=\boldsymbol{r}_{0} \\
\boldsymbol{v}\left(t_{0}\right)=\boldsymbol{v}_{0} \\
m\left(t_{0}\right)=1 \\
\boldsymbol{r}\left(t_{f}\right)=\boldsymbol{r}_{f} \\
\boldsymbol{v}\left(t_{f}\right)=\boldsymbol{v}_{f} .
\end{array}\right.
$$

In an interplanetary transfer, the constraints on the initial and final positions are associated to the initial and final positions of the departure and arrival 
bodies. Thus, once the initial epoch $t_{0}$ and the final epoch $t_{f}$ (or the time of flight $t_{T o F}$, so that $\left.t_{f}=t_{0}+t_{T o F}\right)$ are chosen, $\boldsymbol{r}_{0}$ and $\boldsymbol{r}_{f}$ are available via ephemeris evaluations. As a rendezvous problem is considered here, $\boldsymbol{v}_{f}$ coincides with the velocity of the arrival body, computed with the same ephemeris function. On the other hand, $\boldsymbol{v}_{0}$ can include the contribution given by the launcher, thus $\boldsymbol{v}_{0}=\boldsymbol{v}_{E}\left(t_{0}\right)+\Delta \boldsymbol{v}$, in which $\boldsymbol{v}_{E}\left(t_{0}\right)$ is the velocity of the Earth at the departure epoch.

Through Pontryagin's maximum principle the optimal control problem is reduced to a two-point boundary value problem (TPBVP). First the costate vector $\boldsymbol{\lambda}=\left[\boldsymbol{\lambda}_{r}, \boldsymbol{\lambda}_{v}, \lambda_{m}\right]$ is introduced, together with the Hamiltonian

$$
H=\boldsymbol{\lambda}_{r} \cdot \boldsymbol{v}+\boldsymbol{\lambda}_{v} \cdot\left(-\frac{\mu}{r^{3}} \boldsymbol{r}+c_{1} \frac{u}{m} \boldsymbol{\alpha}\right)-\lambda_{m} c_{2} u+c_{2} u
$$

The optimal thrust direction and magnitude, which minimize the Hamiltonian in Eq. (10), are

$$
\begin{gathered}
\boldsymbol{\alpha}=-\frac{\boldsymbol{\lambda}_{v}}{\lambda_{v}} \\
\begin{cases}u=0 & \text { if } \rho>0 \\
u=1 & \text { if } \rho<0 \\
u \in[0,1] & \text { if } \rho=0,\end{cases}
\end{gathered}
$$

where the switching function $\rho$ is

$$
\rho=1-\frac{c_{1} \lambda_{v}}{c_{2} m}-\lambda_{m}
$$


The dynamics of the costate is given by

$$
\left\{\begin{array}{l}
\dot{\boldsymbol{\lambda}}_{r}=\frac{\mu}{r^{3}} \boldsymbol{\lambda}_{v}-\frac{3 \mu \boldsymbol{r} \cdot \boldsymbol{\lambda}_{v}}{r^{5}} \boldsymbol{r} \\
\dot{\boldsymbol{\lambda}}_{v}=-\boldsymbol{\lambda}_{r} \\
\dot{\boldsymbol{\lambda}}_{m}=-c_{1} \frac{u}{m^{2}} \lambda_{v}
\end{array}\right.
$$

According to the transversality condition, the only constraint for the costate is

$$
\lambda_{m}\left(t_{f}\right)=0,
$$

since the final mass is free.

The optimal control problem is reduced to a TPBVP defined by the dynamics of the state and costate (7) and (14), the optimality conditions (11) and (12), and the constraints (9) and (15). This problem will be referred to as fuel-optimal problem (FOP) in the remainder of the paper. For details on the optimal control problem formulation the reader may refer to Reference 19.

\subsection{Numerical solution of the optimal control problem}

The numerical solution of the TPBV is difficult to obtain because of the small convergence radius, the sensitivity of the initial guesses, the discontinuous integrated functions, and the singular Jacobian matrix. A common practice is to use an homotopic approach by perturbing the performance index with the introduction of a parameter in the range $[0,1]$. The procedure starts with a unitary value of the parameter, for which the numerical solution is easily computed. The FOP is solved by continuously decreasing the perturbation parameter from one to zero and taking the obtained solution as an initial guess for the next iteration [20]. 
An alternative approach, based on a $C^{\infty}$ approximation of the discontinuous optimal control law, is here proposed. The method is based on a three-step procedure and it exhibits high numerical robustness, as $C^{\infty} \mathrm{TP}$ BVP are solved in the first two steps. Furthermore, the second step delivers a solution that is so close to the one of the FOP that numerical convergence is guaranteed.

As a first step, an energy-optimal problem (EOP) is formulated. The constraint on the maximum thrust is removed and the objective function is redefined as

$$
J=\frac{1}{2} \int_{t_{0}}^{t_{f}} a^{2} d t
$$

where $\boldsymbol{a}$ is the acceleration vector given by the thruster. The equations of motion for the spacecraft reduce to

$$
\left\{\begin{array}{l}
\dot{\boldsymbol{r}}=\boldsymbol{v} \\
\dot{\boldsymbol{v}}=-\frac{\mu}{r^{3}} \boldsymbol{r}+\boldsymbol{a} \\
\dot{m}=-\frac{c_{2}}{c_{1}} u m
\end{array}\right.
$$

and the optimality condition to

$$
\boldsymbol{a}=-\boldsymbol{\lambda}_{v}
$$

The mass multiplier is identically zero during the transfer, whereas the dynamics for the position and velocity multipliers read

$$
\left\{\begin{array}{l}
\dot{\boldsymbol{\lambda}}_{r}=\frac{\mu}{r^{3}} \boldsymbol{\lambda}_{v}-\frac{3 \mu \boldsymbol{r} \cdot \boldsymbol{\lambda}_{v}}{r^{5}} \boldsymbol{r} \\
\dot{\boldsymbol{\lambda}}_{v}=-\boldsymbol{\lambda}_{r} .
\end{array}\right.
$$

The set of constraints on the position and the velocity (9) remains unchanged. The TPBVP for the EOP is continuous and its solution can be easily obtained 
by standard nonlinear equations solvers, starting from the initial guess $\boldsymbol{\lambda}_{r}=\mathbf{0}$ and $\boldsymbol{\lambda}_{v}=\mathbf{0}$. The solution of the TPBVP is embedded in a parametric optimization problem, in which $t_{0}, t_{T o F}$, and $\Delta \boldsymbol{v}$ are also optimized. (Note that this aspect can be included in the formulation of the optimal control problem, but this approach has been avoided to keep the TPBVP as simple as possible). A vector of optimization variables is defined as

$$
\boldsymbol{x}=\left[t_{0}, t_{T o F}, \Delta \boldsymbol{v}, \boldsymbol{\lambda}_{r_{0}}, \boldsymbol{\lambda}_{v_{0}}\right]
$$

and the search space is limited by upper and lower bounds (the initial Lagrangian multipliers are left unbounded). The objective function to be minimized is

$$
J=-m_{f}
$$

and the set of constrains are

$$
\left\{\begin{array}{l}
\boldsymbol{r}\left(t_{f}\right)=\boldsymbol{r}_{f} \\
\boldsymbol{v}\left(t_{f}\right)=\boldsymbol{v}_{f} \\
\Delta v \leq \Delta v_{\max }
\end{array}\right.
$$

where $\Delta v_{\max }$ is the maximum value for launcher's contribution. For given arrival body, starting from an initial guess $\boldsymbol{x}_{0}$ (where the initial value for the Lagrangian multipliers is set to zero), the optimization process

1. Computes $\boldsymbol{r}_{0}$ and $\boldsymbol{v}_{0}=\boldsymbol{v}_{E}\left(t_{0}\right)+\Delta \boldsymbol{v}$ at $t_{0}$, and $\boldsymbol{r}_{f}$ and $\boldsymbol{v}_{f}$ at $t_{f}=t_{0}+t_{T 0 F}$ by ephemeris evaluation

2. Integrates the dynamics (17) and (19) from $t_{0}$ to $t_{f}$, with the optimal acceleration profile defined by $\boldsymbol{a}=-\boldsymbol{\lambda}_{v}$

3. Evaluates the objective function (21) and the constraints (22) 
4. Iterates until the constraints are satisfied to a given accuracy and objective function is minimized

A nonlinear quadratic programming method is used for the optimization.

The optimal values found for $\boldsymbol{x}$ are used to initiate the second step of the numerical procedure. In this stage the values of $t_{0}, t_{T o F}$, and $\Delta \boldsymbol{v}$ are frozen, whereas those of the initial Lagrangian multipliers are used as initial guesses of a new optimal control problem. This problem is the same as the FOP, but the discontinuous law for the thrust ratio is approximated by a $C^{\infty}$ function. In particular, an exponential representation is chosen

$$
u=\frac{1}{1+e^{p \rho}}
$$

where $\rho$ is the switching function, and $p$ a continuation parameter. For increasing values of $p$, the continuous representation of $u$ tends to the optimal bang-bang solution of the FOP. The availability of a first guess for the Lagrangian multipliers and the continuous representation of the optimal control law enable the solution of the TPBVP with standard nonlinear equation solvers. A starting value of one for the continuation parameter is usually considered. Then, this value is progressively increased until the expected bang-bang structure appears. Finally, in the last step of the procedure, the TPBVP associated to the FOP is solved with a fifth order finite difference method.

\section{Reference Trajectory}

A rendezvous with asteroid $1996 \mathrm{FG}_{3}$ is considered. This asteroid is a near-Earth minor planet in the Apollo group and it is the primary target 
of the European Space Agency's Marco Polo-R mission. A spacecraft of an initial mass of $1500 \mathrm{~kg}$ is selected with a thrusting capability of $0.33 \mathrm{~N}$ and specific impulse of $3800 \mathrm{~s} . \mathrm{A} t_{0}$ in the interval [4900,5500] MJD2000 is considered and the $t_{T o F}$ is constrained to $[350,550]$ days. A maximum $\Delta v$ of $1 \mathrm{~km} / \mathrm{s}$ is set.

In the EOP, the guesses for $t_{0}$ and $t_{T o F}$ are 5200 MJD2000 and 480 days, respectively. Null initial values for both the $\Delta v$ and the Lagrangian multipliers are used. The optimal solution is characterized by $t_{0}=5314.645$ MJD2000, whereas the $t_{T o F}$ and $\Delta v$ hit their lower and upper bounds, respectively. The mass at rendezvous is $1304.86 \mathrm{~kg}$. The thrust magnitude associated to this solution is plotted with the dotted line in Figure 2(a). Note that the maximum thrust exceeds the thrust available on board, but this is compatible with the EOP formulation.

The solution of the EOP is taken as first guess for the second step. Here both $t_{0}$ and $t_{T o F}$ are fixed, and a sequence of ten TPBVP is solved for increasing values of the continuation parameter. These solutions are plotted with dashed lines in Figure 2(a), where the emergence of the bang-bang structure is clearly visible. The final mass associated to $p=19$ is $1313.26 \mathrm{~kg}$.

In the third step the FOP is addressed. With few iterations, the TPBVP solver computes the optimal discontinuous control law (plotted in Figure $2(\mathrm{a})$ ), which allows for a further increase of the final spacecraft mass up to $1313.68 \mathrm{~kg}$. The projection of the optimal interplanetary transfer in the $x-y$ plane is shown in Figure 2(b). 


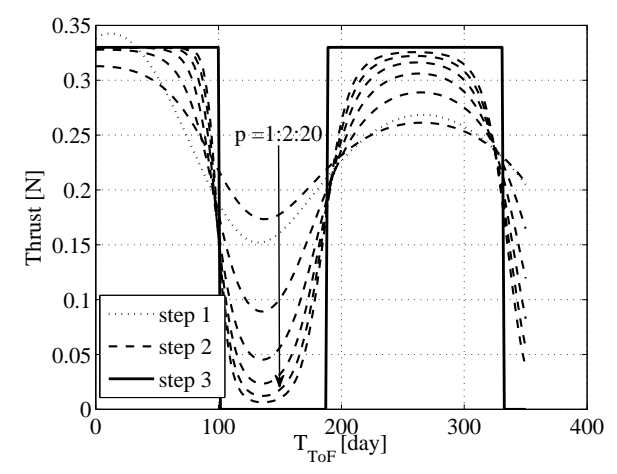

(a) Control magnitude

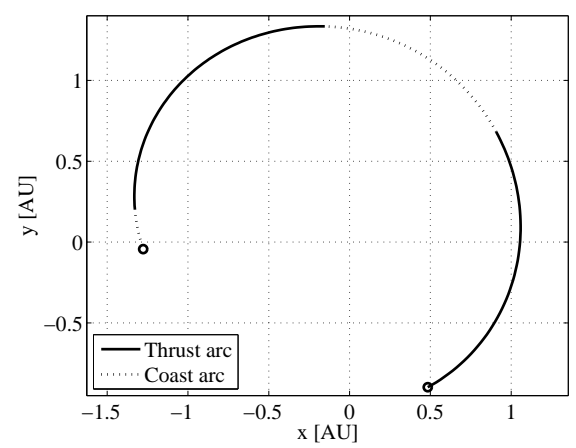

(b) Reference Trajectory

Figure 2: Earth-1996 FG 3 optimal tranfer

\section{DA-based Optimal Feedback Control Algorithm}

Assume a reference solution of the FOP (7)-(9) is available. Thus, the initial costates, $\boldsymbol{\lambda}_{x_{0}}=\left[\boldsymbol{\lambda}_{r_{0}}, \boldsymbol{\lambda}_{v_{0}}\right]$ and $\lambda_{m_{0}}$, the final costates, $\boldsymbol{\lambda}_{x_{f}}=\left[\boldsymbol{\lambda}_{r_{f}}, \boldsymbol{\lambda}_{v_{f}}\right]$ and $\lambda_{m_{f}}$, and the control switching times $t_{s_{i}}, i=1, \ldots, q$, have been computed for assigned initial conditions, $\boldsymbol{x}_{\mathbf{0}}=\left[\boldsymbol{r}_{0}, \boldsymbol{v}_{0}\right]$ and $m_{0}$, and final target position and velocity $\boldsymbol{x}_{\boldsymbol{f}}=\left[\boldsymbol{r}_{f}, \boldsymbol{v}_{f}\right]$. It is worth recalling that $\lambda_{m_{f}}=0$ for the transversality condition. A DA-based algorithm for the arbitrary order expansion of the solution of the FOP with respect to both the initial spacecraft state and the final target state is presented in this section.

Step 1. Initialize the initial spacecraft state, the initial costates, and the first control switching time as DA variables. This means the following 
perturbations with respect to their reference values are considered:

$$
\begin{aligned}
& {\left[\boldsymbol{x}_{0}\right]=\boldsymbol{x}_{0}+\delta \boldsymbol{x}_{0}} \\
& {\left[\boldsymbol{\lambda}_{x_{0}}\right]=\boldsymbol{\lambda}_{x_{0}}+\delta \boldsymbol{\lambda}_{x_{0}}} \\
& {\left[\lambda_{m_{0}}\right]=\lambda_{m_{0}}+\delta \lambda_{m_{0}}} \\
& {\left[t_{s_{1}}\right]=t_{s_{1}}+\delta t_{s_{1}} .}
\end{aligned}
$$

Step 2. Using the DA techniques introduced in Section 3, the ODEs (7) and (14) are integrated from $t_{0}$ to $\left[t_{s_{1}}\right]$ to obtain the high order expansion of the flow. More specifically, the dependence of the spacecraft state, mass, and costates at $\left[t_{s_{1}}\right]$ on the perturbed initial conditions is obtained in terms of the high order polynomial map

$$
\left(\begin{array}{c}
{\left[\boldsymbol{x}_{1}\right]} \\
{\left[m_{1}\right]} \\
{\left[\boldsymbol{\lambda}_{x_{1}}\right]} \\
{\left[\lambda_{m_{1}}\right]}
\end{array}\right)=\left(\begin{array}{c}
\boldsymbol{x}_{1} \\
m_{1} \\
\boldsymbol{\lambda}_{x_{1}} \\
\lambda_{m_{1}}
\end{array}\right)+\left(\begin{array}{l}
\mathcal{M}_{\boldsymbol{x}_{1}} \\
\mathcal{M}_{m_{1}} \\
\mathcal{M}_{\boldsymbol{\lambda}_{x_{1}}} \\
\mathcal{M}_{\lambda_{m_{1}}}
\end{array}\right)\left(\begin{array}{c}
\delta \boldsymbol{x}_{0} \\
\delta \boldsymbol{\lambda}_{x_{0}} \\
\delta \lambda_{m_{0}} \\
\delta t_{s_{1}}
\end{array}\right)
$$

where $\mathcal{M}_{\boldsymbol{x}_{1}}, \mathcal{M}_{m_{1}}, \mathcal{M}_{\boldsymbol{\lambda}_{x_{1}}}$, and $\mathcal{M}_{\lambda_{m_{1}}}$ denote high order polynomials in $\delta \boldsymbol{x}_{0}$, $\delta \boldsymbol{\lambda}_{x_{0}}, \delta \lambda_{m_{0}}$, and $\delta t_{s_{1}}$.

Step 3. Compute the switching function (13) in the DA framework, which yields the Taylor polynomial

$$
\left[\rho_{1}\right]=\delta \rho_{1}=1-\frac{I_{s p} g_{0}\left[\lambda_{v 1}\right]}{\left[m_{1}\right]}-\left[\lambda_{m_{1}}\right]=\mathcal{M}_{\rho_{1}}\left(\delta \boldsymbol{x}_{0}, \delta \boldsymbol{\lambda}_{x_{0}}, \delta \lambda_{m_{0}}, \delta t_{s_{1}}\right)
$$

It is worth observing that the constant part of map (26) is the value of the switching function at $t_{s_{1}}$ for the reference solution, which equals zero. Thus, $\left[\rho_{1}\right]=\delta \rho_{1}$. 
Step 4. Consider the map

$$
\left(\begin{array}{c}
\delta \rho_{1} \\
\delta \boldsymbol{x}_{0} \\
\delta \boldsymbol{\lambda}_{x_{0}} \\
\delta \lambda_{m_{0}}
\end{array}\right)=\left(\begin{array}{c}
\mathcal{M}_{\rho_{1}} \\
\mathcal{I}_{\boldsymbol{x}_{0}} \\
\mathcal{I}_{\boldsymbol{\lambda}_{x_{0}}} \\
\mathcal{I}_{\lambda_{m_{0}}}
\end{array}\right)\left(\begin{array}{c}
\delta \boldsymbol{x}_{0} \\
\delta \boldsymbol{\lambda}_{x_{0}} \\
\delta \lambda_{m_{0}} \\
\delta t_{s_{1}}
\end{array}\right)
$$

which is built by concatenating map (26) with the identity maps for $\delta \boldsymbol{x}_{0}$, $\delta \boldsymbol{\lambda}_{x_{0}}$, and $\delta \lambda_{m_{0}}$.

Step 5. Using polynomial inversion techniques [16], invert map (27) to obtain

$$
\left(\begin{array}{c}
\delta \boldsymbol{x}_{0} \\
\delta \boldsymbol{\lambda}_{x_{0}} \\
\delta \lambda_{m_{0}} \\
\delta t_{s_{1}}
\end{array}\right)=\left(\begin{array}{l}
\mathcal{M}_{\rho_{1}} \\
\mathcal{I}_{\boldsymbol{x}_{0}} \\
\mathcal{I}_{\boldsymbol{\lambda}_{x_{0}}} \\
\mathcal{I}_{\lambda_{m_{0}}}
\end{array}\right)^{-1}\left(\begin{array}{c}
\delta \rho_{1} \\
\delta \boldsymbol{x}_{0} \\
\delta \boldsymbol{\lambda}_{x_{0}} \\
\delta \lambda_{m_{0}}
\end{array}\right)
$$

The fourth component of map (28) relates the first control switching time to the deviation $\delta \rho_{1}$ of the switching function from its reference value $\rho_{1}=0$ and the perturbations $\delta \boldsymbol{x}_{0}, \delta \boldsymbol{\lambda}_{x_{0}}$, and $\delta \lambda_{m_{0}}$.

Step 6. Given any $\delta \boldsymbol{x}_{0}, \delta \boldsymbol{\lambda}_{x_{0}}$, and $\delta \lambda_{m_{0}}$, we can compute the corresponding switching time by imposing $\delta \rho_{1}=0$ in Eq. (28),

$$
\left(\begin{array}{c}
\delta \boldsymbol{x}_{0} \\
\delta \boldsymbol{\lambda}_{x_{0}} \\
\delta \lambda_{m_{0}} \\
\delta t_{s_{1}}
\end{array}\right)=\left(\begin{array}{l}
\mathcal{M}_{\rho_{1}} \\
\mathcal{I}_{\boldsymbol{x}_{0}} \\
\mathcal{I}_{\boldsymbol{\lambda}_{x_{0}}} \\
\mathcal{I}_{\lambda_{m_{0}}}
\end{array}\right)^{-1}\left(\begin{array}{c}
0 \\
\delta \boldsymbol{x}_{0} \\
\delta \boldsymbol{\lambda}_{x_{0}} \\
\delta \lambda_{m_{0}}
\end{array}\right)
$$


Let us extract the fourth component of map (29), which will be indicated as

$$
\delta t_{s_{1}}=\mathcal{M}_{\rho_{1}=0}\left(\delta \boldsymbol{x}_{0}, \delta \boldsymbol{\lambda}_{x_{0}}, \delta \lambda_{m_{0}}\right)
$$

The map (30) can be added to the reference $t_{s_{1}}$ to obtain the Taylor polynomial

$$
\left[t_{s_{1}}\right]_{\rho_{1}=0}=t_{s_{1}}+\delta t_{s_{1}}=t_{s_{1}}+\mathcal{M}_{\rho_{1}=0}\left(\delta \boldsymbol{x}_{0}, \delta \boldsymbol{\lambda}_{x_{0}}, \delta \lambda_{m_{0}}\right)
$$

which is the Taylor expansion of the constraint manifold $\rho_{1}=0$; i.e., the Taylor expansion of the first switching time with respect to the perturbations on the initial conditions.

Step 7. Compose map (25) with (29) to obtain the Taylor expansion of the spacecraft state and costate at the first control switch with respect to the initial conditions,

$$
\left(\begin{array}{c}
{\left[\boldsymbol{x}_{1}\right]} \\
{\left[m_{1}\right]} \\
{\left[\boldsymbol{\lambda}_{x_{1}}\right]} \\
{\left[\lambda_{m_{1}}\right]}
\end{array}\right)_{\rho_{1}=0}=\left(\begin{array}{c}
\boldsymbol{x}_{1} \\
m_{1} \\
\boldsymbol{\lambda}_{x_{1}} \\
\lambda_{m_{1}}
\end{array}\right)+\left(\begin{array}{l}
\mathcal{M}_{\boldsymbol{x}_{1}} \\
\mathcal{M}_{m_{1}} \\
\mathcal{M}_{\boldsymbol{\lambda}_{x_{1}}} \\
\mathcal{M}_{\lambda_{m_{1}}}
\end{array}\right)_{\rho_{1}=0}\left(\begin{array}{c}
\delta \boldsymbol{x}_{0} \\
\delta \boldsymbol{\lambda}_{x_{0}} \\
\delta \lambda_{m_{0}}
\end{array}\right)
$$

The switching time $t_{s_{2}}$ can now be initialized as a DA variable, $\left[t_{s_{2}}\right]=$ $t_{s_{2}}+\delta t_{s_{2}}$, and steps 2 through 7 can be repeated from $\left[t_{s_{1}}\right]_{\rho_{1}=0}$ to $\left[t_{s_{2}}\right]$. By iterating the same procedure till $t_{s_{q}}$ we obtain

$$
\left[t_{s_{q}}\right]_{\boldsymbol{\rho}=0}=t_{s_{q}}+\mathcal{M}_{\boldsymbol{\rho}=0}\left(\delta \boldsymbol{x}_{0}, \delta \boldsymbol{\lambda}_{x_{0}}, \delta \lambda_{m_{0}}\right)
$$


and

$$
\left(\begin{array}{c}
{\left[\boldsymbol{x}_{q}\right]} \\
{\left[m_{q}\right]} \\
{\left[\boldsymbol{\lambda}_{x_{q}}\right]} \\
{\left[\lambda_{m_{q}}\right]}
\end{array}\right)_{\boldsymbol{\rho}=0}=\left(\begin{array}{c}
\boldsymbol{x}_{q} \\
m_{q} \\
\boldsymbol{\lambda}_{x_{q}} \\
\lambda_{m_{q}}
\end{array}\right)+\left(\begin{array}{l}
\mathcal{M}_{\boldsymbol{x}_{q}} \\
\mathcal{M}_{m_{q}} \\
\mathcal{M}_{\boldsymbol{\lambda}_{x_{q}}} \\
\mathcal{M}_{\lambda_{m_{q}}}
\end{array}\right)_{\boldsymbol{\rho}=0}\left(\begin{array}{c}
\delta \boldsymbol{x}_{0} \\
\delta \boldsymbol{\lambda}_{x_{0}} \\
\delta \lambda_{m_{0}}
\end{array}\right)
$$

where $\boldsymbol{\rho}=\left[\rho_{1}, \ldots, \rho_{q}\right]$.

Step 8. A DA-based integration is now performed from $\left[t_{s_{q}}\right]_{\boldsymbol{\rho}=0}$ to $t_{f}$. The resulting polynomial map

$$
\left(\begin{array}{c}
{\left[\boldsymbol{x}_{f}\right]} \\
{\left[m_{f}\right]} \\
{\left[\boldsymbol{\lambda}_{x_{f}}\right]} \\
{\left[\lambda_{m_{f}}\right]}
\end{array}\right)_{\boldsymbol{\rho}=0}=\left(\begin{array}{c}
\boldsymbol{x}_{f} \\
m_{f} \\
\boldsymbol{\lambda}_{x_{f}} \\
\lambda_{m_{f}}
\end{array}\right)+\left(\begin{array}{l}
\mathcal{M}_{\boldsymbol{x}_{f}} \\
\mathcal{M}_{m_{f}} \\
\mathcal{M}_{\boldsymbol{\lambda}_{x_{f}}} \\
\mathcal{M}_{\lambda_{m_{f}}}
\end{array}\right)_{\boldsymbol{\rho}=0}\left(\begin{array}{c}
\delta \boldsymbol{x}_{0} \\
\delta \boldsymbol{\lambda}_{x_{0}} \\
\delta \lambda_{m_{0}}
\end{array}\right)
$$

is the Taylor approximation of the deviation of the final conditions from their reference values caused by the perturbed initial conditions and embedding the satisfaction of $\delta \rho_{i}=0$, for $i=1, \ldots, q$.

Step 9. Extract the first and last components of map (35), subtract their constant parts, and built the following map

$$
\left(\begin{array}{c}
\delta \boldsymbol{x}_{0} \\
\delta \boldsymbol{x}_{f} \\
\delta \lambda_{m_{f}}
\end{array}\right)=\left(\begin{array}{c}
\mathcal{I}_{\boldsymbol{x}_{0}} \\
\mathcal{M}_{\boldsymbol{x}_{f}} \\
\mathcal{M}_{\lambda_{m_{f}}}
\end{array}\right)_{\boldsymbol{\rho}=0}\left(\begin{array}{c}
\delta \boldsymbol{x}_{0} \\
\delta \boldsymbol{\lambda}_{x_{0}} \\
\delta \lambda_{m_{0}}
\end{array}\right),
$$

which includes the identity map on $\delta \boldsymbol{x}_{0}$.

Step 10. Invert map (36) and impose the transversality condition by 
setting $\delta \lambda_{m_{f}}=0$. This yields

$$
\left(\begin{array}{c}
\delta \boldsymbol{x}_{0} \\
\delta \boldsymbol{\lambda}_{x_{0}} \\
\delta \lambda_{m_{0}}
\end{array}\right)=\left(\begin{array}{c}
\mathcal{I}_{\boldsymbol{x}_{0}} \\
\mathcal{M}_{\boldsymbol{x}_{f}} \\
\mathcal{M}_{\lambda_{m_{f}}}
\end{array}\right)^{-1}\left(\begin{array}{c}
\delta \boldsymbol{x}_{0} \\
\delta \boldsymbol{x}_{f} \\
0
\end{array}\right)
$$

Step 11. Add the reference initial costates to the last two components of $(37)$,

$$
\left(\begin{array}{c}
{\left[\boldsymbol{\lambda}_{x_{0}}\right]} \\
{\left[\lambda_{m_{0}}\right]}
\end{array}\right)_{\boldsymbol{\rho}, \lambda_{m_{f}}=0}=\left(\begin{array}{c}
\boldsymbol{\lambda}_{x_{0}} \\
\lambda_{m_{0}}
\end{array}\right)+\left(\begin{array}{l}
\mathcal{M}_{\boldsymbol{\lambda}_{x_{0}}} \\
\mathcal{M}_{\lambda_{m_{0}}}
\end{array}\right)_{\boldsymbol{\rho}, \lambda_{m_{f}}=0}\left(\begin{array}{l}
\delta \boldsymbol{x}_{0} \\
\delta \boldsymbol{x}_{f}
\end{array}\right)
$$

and compose map (31) and its counterparts for the subsequent switching times with (37) to obtain

$$
\left[t_{s_{i}}\right]_{\boldsymbol{\rho}, \lambda_{m_{f}}=0}=t_{s_{i}}+\mathcal{M}_{\boldsymbol{\rho}, \lambda_{m_{f}}=0}\left(\delta \boldsymbol{x}_{0}, \delta \boldsymbol{x}_{f}\right)
$$

for $i=1, \ldots, q$. Together, maps (38) and (39) identify the high order Taylor expansion of the solution of the optimal control problem with respect to the initial spacecraft state and the final target state. More specifically, for any $\delta \boldsymbol{x}_{0}$ and $\delta \boldsymbol{x}_{f}$, the mere evaluation of the polynomial map (38) delivers the associated optimal costates. In addition, the polynomials (39) can be evaluated to identify the corresponding optimal control switching times. It is worth observing that the polynomial maps (38) and (39) supply high order Taylor approximations of the solution of the optimal control problem for perturbed initial and final conditions, which are accurate up to the order used for the DA-based computation. 


\section{High Order Optimal Feedback of the Earth-1996 FG $_{3}$ transfer}

The performances of the DA-based optimal feedback control algorithm are assessed on the Earth-1996 $\mathrm{FG}_{3}$ transfer introduced above. The fuel optimal transfer trajectory and the control profile reported in Figures 2(b) and 2(a) are used as reference for the expansion of the solution of the FOP. The algorithm introduced in the previous section is then applied to compute a fourth order Taylor expansion of the solution of the FOP about the reference trajectory. Thus, the resulting maps (38) and (39) are fourth order polynomials in $\delta \boldsymbol{x}_{0}$ and $\delta \boldsymbol{x}_{f}$. The case of perturbed final target positions is first investigated. The analysis for perturbed initial spacecraft positions follows.

\subsection{Perturbed final target positions}

The final position of asteroid $1996 \mathrm{FG}_{3}, \boldsymbol{r}_{f}$, is now supposed to be affected by measurable errors. Given any displacement $\delta \boldsymbol{r}_{f}$ of the final target position from its reference value, the polynomial map (38) is evaluated by setting $\delta \boldsymbol{x}_{0} \equiv\left[\delta \boldsymbol{r}_{0}, \delta \boldsymbol{v}_{0}\right]=[0,0]$ and $\delta \boldsymbol{x}_{f} \equiv\left[\delta \boldsymbol{r}_{f}, \delta \boldsymbol{v}_{f}\right]=\left[\delta \boldsymbol{r}_{f}, 0\right]$. The corresponding optimal values of $\boldsymbol{\lambda}_{x_{0}}$ and $\lambda_{m_{0}}$ are computed. Then, starting from the reference initial spacecraft state and the new initial costates, a forward pointwise integration of the ODEs (7) and (14) supplies the optimal control law to transfer the spacecraft from the reference initial state to the perturbed final target position $\tilde{\boldsymbol{r}}_{f}=\boldsymbol{r}_{f}+\delta \boldsymbol{r}_{f}$. Moreover, map (39) is evaluated to identify the optimal control switching times of the new trajectory.

The performances of the procedure are studied hereafter. A maximum position error of 1E-3 AU is supposed to affect each component of the final target state. For each corner of the corresponding cube, the associated $\delta \boldsymbol{r}_{f}$ is 


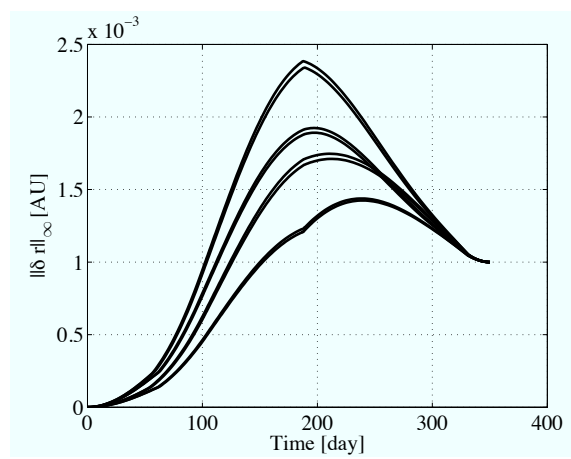

(a) Trajectory displacement along the transfer

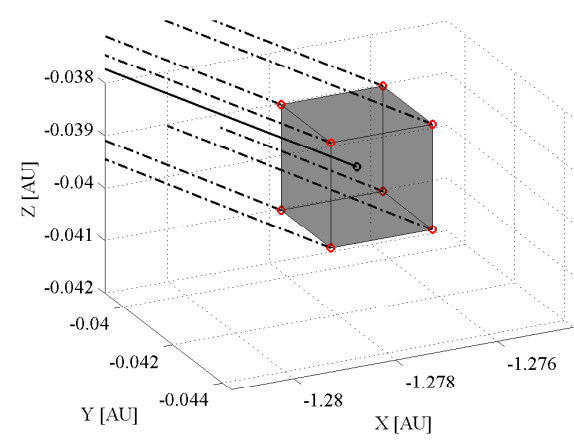

(b) Detail at arrival

Figure 3: Earth-1996 $\mathrm{FG}_{3}$ transfer: optimal feedback on perturbed final target positions.

computed and the map (38) is evaluated to obtain the new optimal transfer trajectory. For each sample, the maximum norm of the difference between the resulting trajectory $\tilde{\boldsymbol{r}}(t)$ and the reference trajectory $\boldsymbol{r}(t)$ is reported in Figure 3(a). Starting from the reference initial position, the new trajectories tend to move away from the reference along the transfer and reach the final imposed displacement of 1E-3 AU. A detail of all trajectories at arrival is reported in Figure 3(b). Thanks to the high order feedback, each new optimal trajectory hits the corresponding perturbed target.

For each sample, map (39) is evaluated to obtain the corresponding optimal control switching times. Figure 4(a) plots the resulting optimal control magnitude profiles, whereas a detail on the last control switch is reported in Figure 4(b). The optimal switching time for the sampled trajectories varies within a range of about 2 days. Once again, it is worth highlighting that the computation of each optimal switching time is reduced to the mere evaluation of a polynomial. 


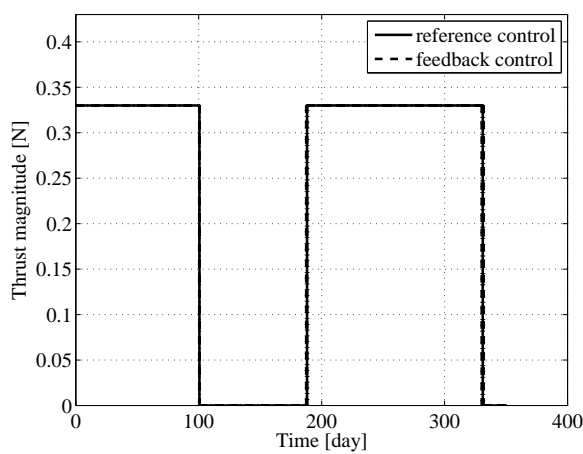

(a) Control profiles

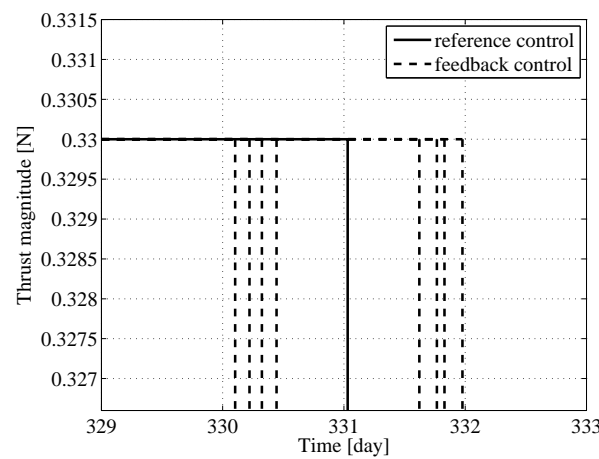

(b) Detail on last control switch

Figure 4: Optimal control profiles for perturbed final target positions.

The accuracy of the fourth order maps (38) and (39) is investigated in Figure $6(\mathrm{a})$. More specifically, the difference $\delta \tilde{\boldsymbol{r}}_{f}$ between the final position and the desired perturbed final target position is computed (refer to Figure 5 for an illustration of the defined quantities). The maximum norm for all samples is then reported. The same process is repeated for different error box amplitudes. As expected, the error increases with box amplitude. Considering $0.1 \%$ as an adequate maximum relative error in the fulfillment of the boundary constraints, Figure 6(a) shows that the fourth order maps are not adequate to compute control corrections for error boxes of amplitude larger than 1E-3 AU. However, this value is well above the errors to be managed in practical applications, as an adequate autonomous navigation systems can guarantee a maximum position error of the order of $100 \mathrm{~km}$ [21]. It is worth observing that the oscillation of the error in Figure 6(a) for box amplitudes smaller than about 1E-4 AU is due to the tolerance of the eight order Runge-Kutta-Fehlberg integration scheme used to integrate equations (7) and (14) and to obtain the fourth order Taylor expansion of their flow. 


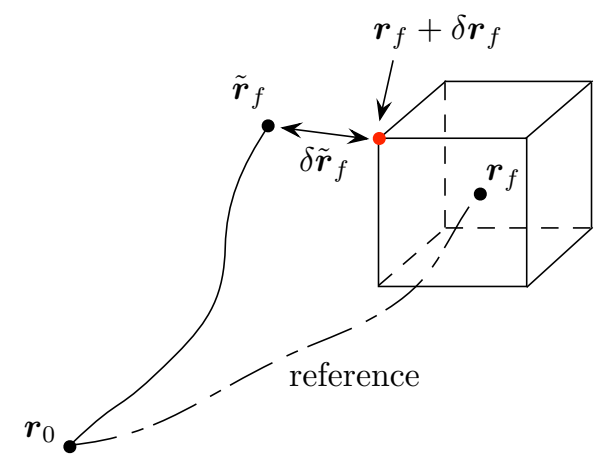

Figure 5: Illustration of the transfer to a perturbed final target position.

Thus, the integration error and, consequently, $\delta \tilde{\boldsymbol{r}}_{f}$ tend to have an irregular behavior below $10^{-12} \mathrm{AU}$.

The effects of the order on the accuracy of the Taylor expansions is studied in Figure $6(\mathrm{~b})$. The trend of $\delta \tilde{\boldsymbol{r}}_{f}$ is reported in figure for increasing expansion orders and different error box amplitudes. The accuracy of the maps increases with order for box amplitudes up to 1E-3 AU and fourth order maps are able to guarantee a maximum relative error of $0.1 \%$ for a box amplitude of $1 \mathrm{E}-3$ $\mathrm{AU}$ on the final target position. The same accuracy is reached with second order maps for an error box amplitude of 1E-4 AU.

Unlike the 1E-4 AU and 1E-3 AU cases, the magnitude of $\delta \tilde{\boldsymbol{r}}_{f}$ does not decrease monotonically with order for a box amplitude of 1E-2 AU. This trend reveals that the Taylor expansions do not converge for some $\delta \boldsymbol{r}_{f}$ between $1 \mathrm{E}$ $3 \mathrm{AU}$ and 1E-2 AU; i.e., the convergence radius of the Taylor expansions lies in the interval [1E-3, 1E-2] AU and, within this interval, increasing the order does not necessarily decrease the error in the fulfillment of the boundary constraints. 


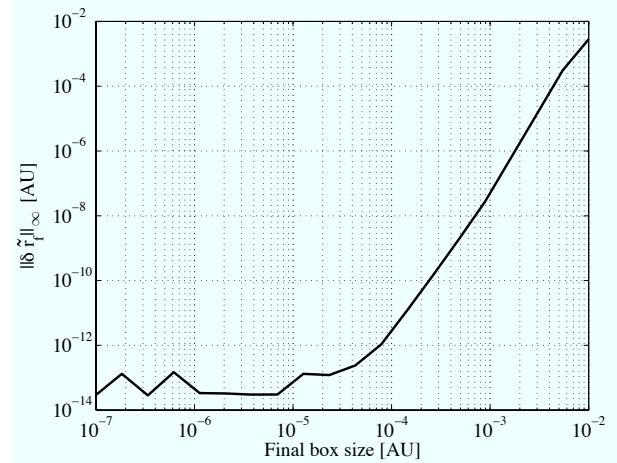

(a) Error vs. Box amplitude

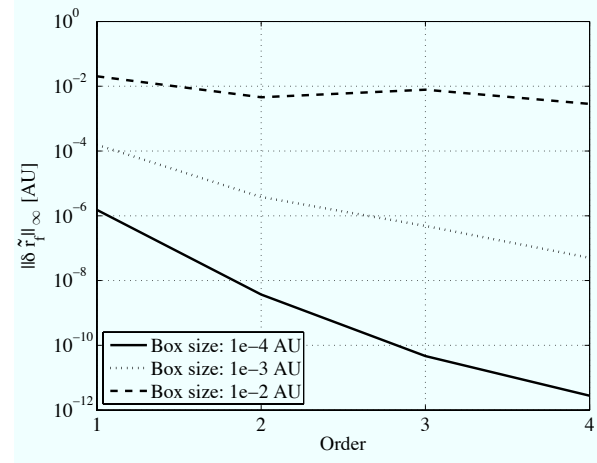

(b) Error vs. Order

Figure 6: Accuracy analysis for perturbed final target positions.

The rationale behind the lack of convergence of the Taylor expansions for error boxes of amplitude larger than 1E-3 AU is investigated in Figure 7(a) and Figure 7(b). More specifically, the two figures report the switching function profiles computed for each corner of the error box, considering box amplitudes of 1E-3 AU and 1E-2 AU, respectively. As can be seen from Figure 7(a), the variation of the switching function for a box amplitude of $1 \mathrm{E}-3 \mathrm{AU}$ is relatively small: the slight change of the optimal control switching times is well described by the fourth order maps and the overall structure of the bang-bang control profile (e.g., number of bangs and control switch location) does not break. This is not the case for a box amplitude of 1E-2 AU. Figure 7 (b) shows that the switching function reaches zero at the end of the transfer for some samples, which warns of the appearance of a new switch. However, the algorithm presented in Section 6 works only for a given structure of the bang-bang control profile: the appearance of additional control switches is a discontinuity that can not be handled by the Taylor expansion. 


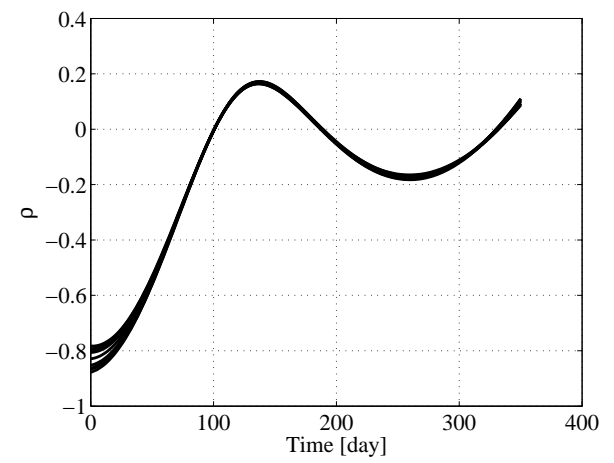

(a) Box amplitude: $10^{-3} \mathrm{AU}$

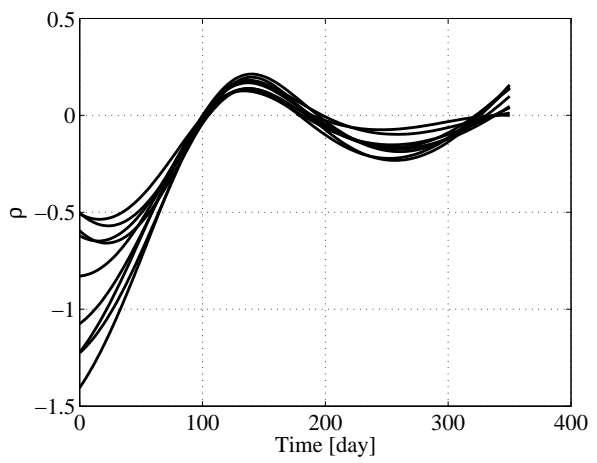

(b) Box amplitude: $10^{-2} \mathrm{AU}$

Figure 7: Switching function profiles for perturbed final target positions.

\subsection{Perturbed initial spacecraft positions}

Similarly to the previous section, the case of perturbed initial spacecraft positions is now analyzed. The vector $\boldsymbol{r}_{0}$, is supposed to be affected by measurable errors. Given any $\delta \boldsymbol{r}_{0}$, the polynomial map (38) is evaluated at $\delta \boldsymbol{x}_{0}=\left[\delta \boldsymbol{r}_{0}, 0\right]$ and $\delta \boldsymbol{x}_{f}=[0,0]$. That is, for any perturbed initial position, the optimal values of $\boldsymbol{\lambda}_{x_{0}}$ and $\lambda_{m_{0}}$ to reach the reference position of $1996 \mathrm{FG}_{3}$ at $t_{f}$ are computed. Moreover, map (39) is evaluated to identify the optimal control switching times of the new trajectory.

As in the previous test case, a maximum position error of 1E-3 AU is supposed to affect each component of the initial spacecraft position. The corners of the associated cube are sampled and the map (38) is evaluated for the optimal correction of the initial costates and control profiles. For each sample, the maximum norm between the resulting trajectories $\tilde{\boldsymbol{r}}(t)$ and the reference trajectory $\boldsymbol{r}(t)$ is reported in Figure 8. Starting from the initial displacement of 1E-3 AU, the new trajectories approach the reference one along the transfer and cancel the error at $t_{f}$. 


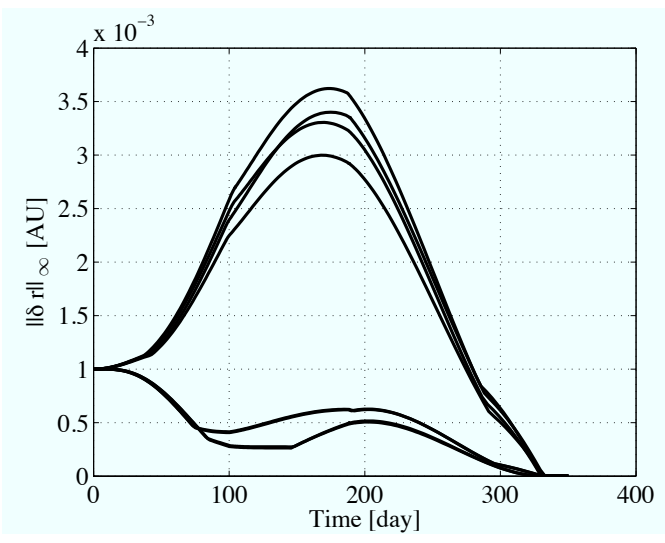

Figure 8: Earth-1996 $\mathrm{FG}_{3}$ transfer: optimal feedback on perturbed initial spacecraft positions. Trajectory displacement along the transfer.

Map (39) is again evaluated to obtain the optimal control switching times for the perturbed trajectories. The resulting Taylor polynomial in $\delta \boldsymbol{r}_{0}$ is reported in Appendix A for illustrative purpose. Figure 9(a) plots the resulting optimal control magnitude profiles, whereas Figure 9(b) reports a detail on the resulting first control switch. The effect of the perturbed initial spacecraft positions on the control profiles is greater than in the case of perturbed final target positions, and the optimal switching time for the sampled trajectories varies within a range of about 8 days.

Finally, the accuracy of the fourth order maps (38) and (39) is investigated by computing the final position error $\delta \boldsymbol{r}_{f}$ with respect to the target (refer to Figure 10(a) for an illustration of the defined quantities). The maximum norm over all samples is reported in Figure 10(b) for different error box amplitudes. The error increases with box amplitude. A comparison between Figure 10(b) and Figure 6(a) shows that the maps (38) and (39) are less accurate when used for the optimal feedback on the initial position. Nevertheless, 


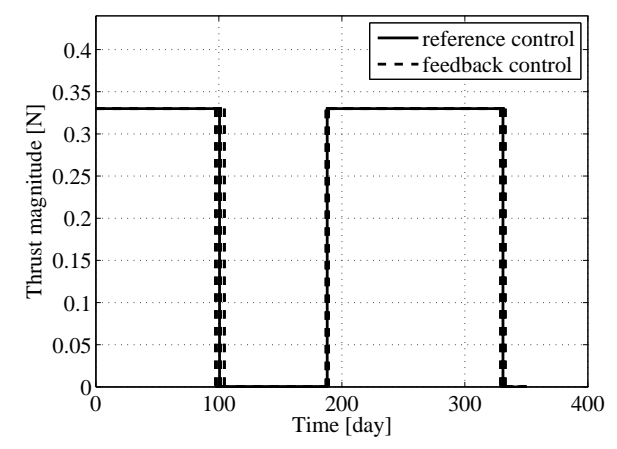

(a) Control profiles

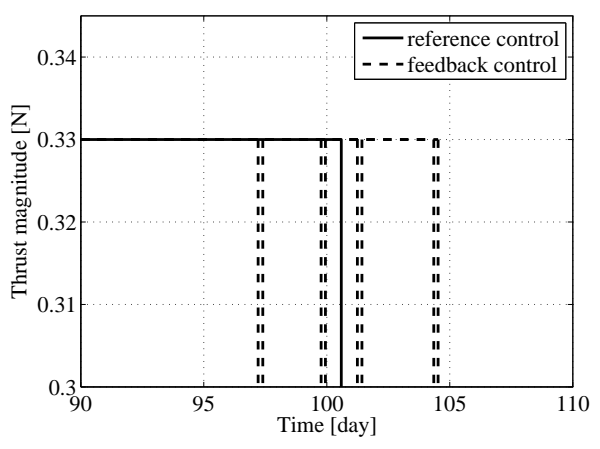

(b) Detail on first control switch

Figure 9: Optimal control profiles for perturbed initial spacecraft positions.

similarly to the case of perturbed final positions, the maps are sufficiently accurate to compute control corrections for error boxes of amplitude up to 1E-3 AU.

\section{Conclusion}

A method for the computation of optimal feedback control laws with saturating actuators based on differential algebra has been introduced, with applications to a transfer to the asteroid $1996 \mathrm{FG}_{3}$. The method relies on the high order expansion of the solution of the optimal control problem about a reference trajectory, which is obtained in terms of arbitrary order Taylor polynomials for the initial costates and the control switching times. Thus, it improves the results of classical techniques based on the linearization of the dynamics. Moreover, it reduces the computation of new optimal control laws to the mere evaluation of polynomials, which is a valuable advantage over the conventional nonlinear optimal control strategies that are mainly based on iterative procedures. The solution of the optimal control problem has 


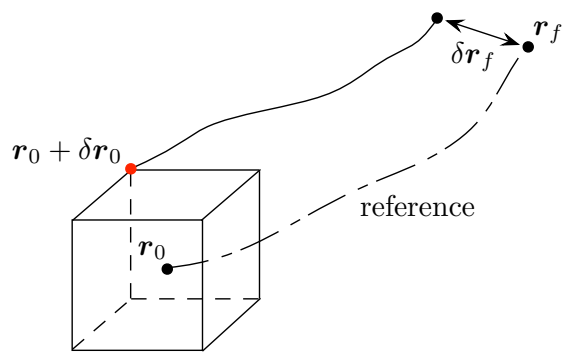

(a) Definition of the quantities for accuracy analysis

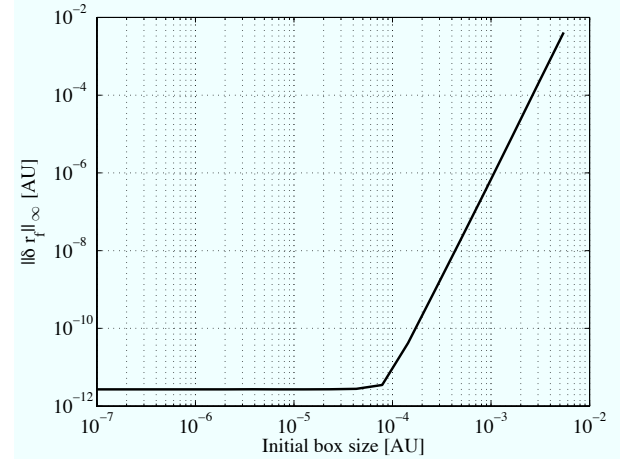

(b) Error vs. Box amplitude

Figure 10: Accuracy analysis for perturbed initial spacecraft positions.

been expanded with respect to both the initial and final boundary conditions, allowing the method to manage 1E-3 AU error boxes on the initial spacecraft and final target positions: using fourth order Taylor expansions, the method is able to meet boundary constraints with a maximum absolute error of $1 \mathrm{E}$ 6 AU, i.e., a maximum relative error of $0.1 \%$. For larger error boxes, the accuracy of the Taylor expansions drops off rapidly. This was shown to be related to the appearance of additional switches in the control law.

The paper presented high order feedback control laws for uncertain initial and final conditions. The same approach can be applied to manage errors at any time along the transfer and, thus, it is suitable for the design of mid-course maneuvers. To this aim, suppose $t_{M C}$ is the time of the midcourse maneuver required to reach the desired target at time $t_{f}$. Suppose that the nominal reference trajectory from $t_{M C}$ to $t_{f}$ is available. The algorithm introduced can be applied to Taylor expand the solution of the optimal control problem from $t_{M C}$ to $t_{f}$ about the reference trajectory. Thus, given 
the propagated or measured error at $t_{M C}$, the Taylor polynomials can be readily evaluated to compute the new optimal control law and to design the associated trajectory from $t_{M C}$ to $t_{f}$.

This work focused on the problem of transferring a spacecraft from an initial fixed state to a final fixed state. Further developments will investigate the imposition of soft constraints, as well as the minimization of alternative performance criteria. In addition, the performances of the method will be assessed on transfers involving an increasing number of control switches.

\section{Acknowledgment}

This research has been carried out in collaboration with the Advanced Concept Team of the European Space Agency under the ESA/Ariadna scheme. The authors are grateful to Dario Izzo for his support. Moreover, the authors are indebted to Martin Berz and Kyoko Makino for having introduced us to differential algebraic techniques as well as for the implementation of COSY-Infinity, without which this work would have not been possible.

\section{Appendix A}

An example of the polynomial maps used for the optimal feedback on perturbed initial spacecraft positions is illustrated hereafter. Table .1 reports the fourth order Taylor approximation of the optimal variation of the first control switching time $\left[t_{s, 1}\right]$ for the case of perturbed initial spacecraft positions. It is worth reporting that the Taylor polynomials are obtained in the scaled vector $\delta \boldsymbol{r}_{0}^{*}=1 \mathrm{E}-4 \delta \boldsymbol{r}_{0}$ to favor the floating point representation of the coefficients. 
Table .1: Polynomial map for the first optimal control switching time of the perturbed initial spacecraft positions test case.

\begin{tabular}{|c|c|c|c|c|c|c|c|c|c|}
\hline \multirow[t]{2}{*}{ Coefficient } & \multirow[t]{2}{*}{ Order } & \multicolumn{3}{|c|}{ Exponents } & \multirow[t]{2}{*}{ Coefficient } & \multirow[t]{2}{*}{ Order } & \multicolumn{3}{|c|}{ Exponents } \\
\hline & & $\delta r_{0, x}^{*}$ & $\delta r_{0, y}^{*}$ & $\delta r_{0, z}^{*}$ & & & $\delta r_{0, x}^{*}$ & $\delta r_{0, y}^{*}$ & $\delta r_{0, z}^{*}$ \\
\hline 1.74264155 & 0 & 0 & 0 & 0 & $-0.19121658 \mathrm{E}-08$ & 3 & 1 & 0 & 2 \\
\hline $0.10351801 \mathrm{E}-01$ & 1 & 1 & 0 & 0 & $0.25879864 \mathrm{E}-08$ & 3 & 0 & 1 & 2 \\
\hline $0.37132630 \mathrm{E}-02$ & 1 & 0 & 1 & 0 & $0.77915643 \mathrm{E}-10$ & 3 & 0 & 0 & 3 \\
\hline$-0.15852277 \mathrm{E}-03$ & 1 & 0 & 0 & 1 & 0.10324371E-09 & 4 & 4 & 0 & 0 \\
\hline $0.12206882 \mathrm{E}-04$ & 2 & 2 & 0 & 0 & $-0.43691257 \mathrm{E}-09$ & 4 & 3 & 1 & 0 \\
\hline$-0.23635223 \mathrm{E}-04$ & 2 & 1 & 1 & 0 & 0.68719026E-09 & 4 & 2 & 2 & 0 \\
\hline $0.11904053 \mathrm{E}-04$ & 2 & 0 & 2 & 0 & $-0.47793450 \mathrm{E}-09$ & 4 & 1 & 3 & 0 \\
\hline$-0.51773789 \mathrm{E}-06$ & 2 & 1 & 0 & 1 & $0.12456629 \mathrm{E}-09$ & 4 & 0 & 4 & 0 \\
\hline $0.13690460 \mathrm{E}-06$ & 2 & 0 & 1 & 1 & $0.15730715 \mathrm{E}-10$ & 4 & 3 & 0 & 1 \\
\hline $0.10597397 \mathrm{E}-05$ & 2 & 0 & 0 & 2 & $-0.42300134 \mathrm{E}-10$ & 4 & 2 & 1 & 1 \\
\hline$-0.39298703 \mathrm{E}-07$ & 3 & 3 & 0 & 0 & $0.37990436 \mathrm{E}-10$ & 4 & 1 & 2 & 1 \\
\hline $0.11734948 \mathrm{E}-06$ & 3 & 2 & 1 & 0 & $-0.11372453 \mathrm{E}-10$ & 4 & 0 & 3 & 1 \\
\hline$-0.11907183 \mathrm{E}-06$ & 3 & 1 & 2 & 0 & $0.14515782 \mathrm{E}-11$ & 4 & 2 & 0 & 2 \\
\hline $0.40951608 \mathrm{E}-07$ & 3 & 0 & 3 & 0 & $-0.93897142 \mathrm{E}-12$ & 4 & 1 & 1 & 2 \\
\hline$-0.98242417 \mathrm{E}-09$ & 3 & 2 & 0 & 1 & $-0.27550454 \mathrm{E}-11$ & 4 & 0 & 2 & 2 \\
\hline $0.11137938 \mathrm{E}-08$ & 3 & 1 & 1 & 1 & $0.26548696 \mathrm{E}-11$ & 4 & 1 & 0 & 3 \\
\hline \multirow[t]{2}{*}{$0.30284059 \mathrm{E}-09$} & 3 & 0 & 2 & 1 & $-0.48321846 \mathrm{E}-11$ & 4 & 0 & 1 & 3 \\
\hline & & & & & $0.90833866 \mathrm{E}-13$ & 4 & 0 & 0 & 4 \\
\hline
\end{tabular}




\section{References}

[1] B. Bouchard, R. Elie, C. Imbert, Optimal Control under Stochastic Target Constraints, SIAM J. Control Optim., 48 (2010) 3501-3531.

[2] M. Vasile, Robust Optimisation of Trajectories Intercepting Dangerous NEO, AIAA/AAS Astrodynamic Specialist Conference, (2002) AIAA Paper 4719.

[3] R.E. Bellman, S.E. Dreyfus, Applied Dynamic Programming. Princeton, N.J., 1962.

[4] R.C. Durbeck, An approximation technique for suboptimal control, IEEE Transactions on Automatic Control, 10 (1965) 144-149.

[5] R. Beard, G. Saridis, J. Wen, Galerkin approximation of the Generalized Hamilton-Jacobi-Bellman equation. Automatica 33(1997) 2159-2177.

[6] J.D. Pearson, Approximation Methods in Optimal Control, Journal of Electronics and Control, 13 (1962) 453-465.

[7] J.A. Burghart, A Technique for Suboptimal Control of Nonlinear Systems, IEEE Transactions on Automatic Control, 14 (1969) 530-533.

[8] S.C. Beeler, State-dependent Riccati Equation Regulation of Systems with State and Control Nonlinearities, NIA Report No. 2004-08, National Institute of Aerospace, Hampton, Virginia, 2004.

[9] C. Park, D. Scheeres, Solutions of the optimal feedback control problem using Hamiltonian dynamics and generating functions, Proceedings of the 42nd IEEE Conference on Decision and Control, 2 (2003) 1222-1227. 
[10] J.A. Fax, R.M. Murray, Finite-horizon optimal control and stabilization of time-scalable systems, Proceedings of the 39st IEEE Conference on Decision and Control, (2000) 748-753.

[11] T. Cimen, S. Banks, Nonlinear Optimal Tracking Control with Application to Super-Tankers for Autopilot Design, Automatica, 40 (2004) 1845-1863.

[12] C. Park, D. Scheeres, Solution of Optimal Feedback Control Problems with General Boundary Conditions using Hamiltonian Dynamics and Generating Functions, Automatica,42 (2006) 869-875.

[13] C. Mracek, J. Cloutier, Control Designs for the Nonlinear Benchmark Problem via the State-dependent Riccati Equation Method, Int. J. Robust Nonlinear Control, 8 (1998) 401-433.

[14] J. Cloutier, C. D'Souza, C. Mracek, Nonlinear Regulation and Nonlinear $\mathrm{H}_{\infty}$ Control via the State-dependent Riccati Equation Technique; Part 1, theory; Part 2, examples, Proceedings of the First International Conference on Nonlinear Problems in Aviation and Aerospace, (1996) $117-141$.

[15] C. Park, D. Scheeres, Extended Applications of Generating Functions to Optimal Feedback Control Problems, Proceedings of the American Control Conference, 2 (2005) 852.

[16] M. Berz, Modern Map Methods in Particle Beam Physics, Academic Press, New York, 1999. 
[17] M. Berz, K. Makino, COSY INFINITY version 9 reference manual, MSU Report MSUHEP-060803, Michigan State University, East Lansing, MI, 2006.

[18] P. Di Lizia, R. Armellin, F. Bernelli-Zazzera, M. Berz, High Order Optimal Feedback Control of Lunar Landing and Rendezvous Trajectoriesl, Proceedings of the 3rd CEAS Air \& Space Conference and XXI Congresso Nazionale AIDAA, (2011) 1221-1230.

[19] A. Bryson, Y. Ho, Applied Optimal Control: optimization, estimation, and control, Hemisphere Publishing Co.s, Washigton, 1975.

[20] F. Jiang, H. Baoyin, J. Li, Practical Techniques for Low-Thrust Trajectory Optimization with Homotopic Approachs, Journal of Guidance, Control, and Dynamics, 35 (2012) 245-258.

[21] J.E. Riedel, S. Desai, D. Han, B. Kennedy, G.W. Null, S.P. Synnott, T.C. Wang, R.A. Werner, E.B. Zamani, Autonomous optical navigation (AutoNav) DS1 technology validation report, Jet Propulsion Laboratory, 2001. 